\title{
A new kind of heterodyne measurement of Coherent Population Trapping in an atomic beam
}

\author{
G. Müller ${ }^{\mathrm{a}}, \mathrm{A}$. Wicht ${ }^{\mathrm{a}}$, R. Rinkleff ${ }^{\mathrm{a}}, \mathrm{K}$. Danzmann ${ }^{\mathrm{a}, \mathrm{b}}$ \\ anstitut für Atom- und Molekülphysik, Universität Hannover, Appelstr. 2, D-30167 Hannover, Germany \\ h Max Planck Institut für Quantenoptik, Außenstelle Hannover, Appelstr. 2, D-30167 Hannover, Germany
}

Received 27 July 1995; revised version received 2 January 1996; accepted 5 February 1996

\begin{abstract}
A new heterodyne technique is presented for the simultaneous measurement of dispersion and absorption of atomic transitions useful especially for coherent two photon transitions. This technique is compared with traditional homodyne interferometer and with modern frequency modulation techniques. First results are the properties of a coherent population trapping (CPT) scheme realized in a cesium atomic beam. The measured linewidth of the CPT-resonance is $\simeq 300 \mathrm{kHz}$ (FWHM) with a residual absorption of less than $2 \times 10^{-3} \mathrm{~cm}^{-1}$ and the dispersion is equivalent to a group velocity of $l \mathrm{~g} \approx \mathrm{c} / 5000$.
\end{abstract}

In the last few years a lot of theoretical work has been done analyzing coherent effects in three-level atoms coupled with two laser fields [1,2]. Possible applications have been discussed as a novel atomic magnetometer [3] or as a tool to increase the sensitivity of an interferometer like a gravitational wave detector [4]. A number of experiments were done measuring the absorption and dispersion with a MachZehnder interferometer, in a gas cell $[5,6]$. Other groups have investigated the fluorescence in an atomic beam [7] without directly measuring the dispersion. These techniques to experimentally characterize coherent effects in atoms are based on comparing two optical lengths with one laserficld (homodync detcctor) and are plagued by inherent problems like vibration or acoustic noise which limit the sensitivity of any interferometer using only one field $[5,6]$. Another possibility to measure dispersion and absorption of an atomic transition is the frequency modulation spectroscopy (FMS) described in Refs. [8,9]. This technique is based on comparing the optical lengths of different frequency components of a frequency modulated field. Although this technique is insensitive to acoustic noise, the signal to shot noise ratio is reduced due to a value proportional to the modulation index which is small compared with unity $(M \ll 1)$.

In this letter a new possibility similar to FMS with $M$ in the order of 1 will be described to measure the dispersion and absorption of an optical atomic transition simultaneously, avoiding most of the traditional problems. This setup is orders of magnitude less sensitive to acoustic noise or vibrations as the homodyne interferometer with a signal strength as high as in the homodyne setup. Especially it is convenient for coherent two photon transitions with two different coherent fields in the interaction region. As an example, the re- 


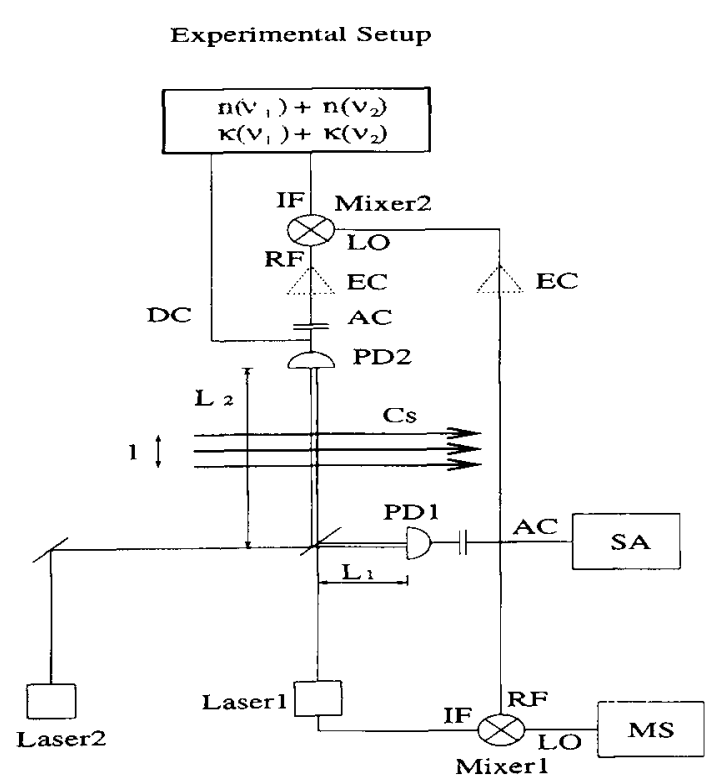

Fig. 1. Both lasers are superimposed at the beamsplitter. The beat signal is recorded first before entering the interaction zone (optical length: $L_{1}$ ) and second behind the interaction zone (optical length: $L_{2}+\delta \mathrm{n} 1$ ). Comparing these beat signals with mixer 2 , the index of refraction is contained in the phase of the IF-signal. MS: microwave synthesizer; SA: spectrum analyzer; RF; reference frequency; LO: local oscillator; IF: intermediate frequency; EC: electronic comparator; Cs: cesium atomic beam

sults of the first measurement of these properties in a CPT-resonance in an atomic beam of Cs will be presented.

The technique is based on a common-path heterodyne interferometer where the pump beam superimposed on the probe beam is also used as the reference beam. The reduction of the noise in the signal induced by vibrations is achieved by recording the beat signal of both lasers with fast photodetectors first before and second after the interaction zone. The second beat depends on the absorption and dispersion in the probe. The absorption in the probe is given by the DC-signal of the photodetector. The induced phaseshift in the second beat could now be demodulated with the first beat.

The experimental setup is shown in Fig. 1. Two laser bcams arc supcrimposed at a beamsplitter. One output is focused on a fast photodetector (PD1) to detect the beat signal between both lasers:

$$
\begin{aligned}
I_{\mathrm{PD} 1}=\eta_{1} \mid & E_{10} \cos \left[\omega_{1}\left(t+L_{1} / c\right)\right] \\
& +\left.E_{20} \cos \left[\omega_{2}\left(t+L_{1} / c\right)\right]\right|^{2} \\
=\eta_{1} & \left\{\left(I_{10}+I_{20}\right)\right. \\
& \left.+\frac{1}{2} E_{10} E_{20} \cos \left[\left(\omega_{1}-\omega_{2}\right)\left(t+L_{1} / c\right)\right]\right\} \\
=I_{\mathrm{DC} 1} & +I_{\mathrm{ACl}},
\end{aligned}
$$

where $\eta_{1}$ is the efficiency of PD1 and $I_{i 0}=\frac{1}{2} E_{i 0}^{2}$ the power of laser $i$. The phase of this signal depends on the distance $L_{1}$ between the pholodetector and the beamsplitter. One part of this signal is connected to the local oscillator ( $\mathrm{LO}$ ) entrance of mixer 2 (the dashed lined electronic comparators (EC) will be described later). The other part of the signal is given on the reference frequency entrance (RF) of mixer 1 and on a spectrum analyzer (SA) to control the difference frequency of the lasers. The LO-entrance of mixer 1 is connected to a tunable microwave-synthesizer (MS). The intermediate frequency (IF)-output of mixer 1 is used to synchronize the phase of laser 1 with that of laser 2 at a difference frequency generated by the synthesizer. With this setup the frequency stability of the difference frequency is equal to the frequency stability of the MS [10].

The second output of the beamsplitter goes through the probe, an atomic beam or a gas cell, for example. Behind the interaction zone the light is focused on the second fast photodetector (PD2, efficiency $\eta_{2}$ ):

$$
\begin{aligned}
I_{\mathrm{PD} 2} & =\eta_{2} \mid E_{10} \mathrm{e}^{-\alpha_{1}\left(\omega_{1}\right) l / 2} \\
& \times \cos \left[\omega_{1}\left(t+L_{2} / c\right)+\delta n_{1}\left(\omega_{1}\right) \omega_{1} l / c\right] \\
& +E_{20} \mathrm{e}^{-\alpha_{2}\left(\omega_{2}\right) l / 2} \\
& \times\left.\cos \left[\omega_{2}\left(t+L_{2} / c\right)+\delta n_{2}\left(\omega_{2}\right) \omega_{2} l / c\right]\right|^{2} \\
= & \eta_{2}\left(I_{10} \mathrm{e}^{-\alpha_{1}\left(\omega_{1}\right) l}+I_{20} \mathrm{e}^{-\alpha_{2}\left(\omega_{2}\right) l}\right) \\
+ & \frac{1}{2} \eta_{2} E_{10} E_{20} \mathrm{e}^{-\left|\alpha_{1}\left(\omega_{1}\right)+\alpha_{2}\left(\omega_{2}\right)\right| t / 2} \\
\times & \cos \left\{\left(\omega_{1}-\omega_{2}\right)\left(t+L_{2} / c\right)\right. \\
& \left.\quad+\left[\delta n_{1}\left(\omega_{1}\right) \omega_{1}-\delta n_{2}\left(\omega_{2}\right) \omega_{2}\right] l / c\right\} \\
= & I_{\mathrm{DC} 2}\left[\alpha_{1}\left(\omega_{1}\right), \alpha_{2}\left(\omega_{2}\right)\right] \\
& +I_{\mathrm{AC} 2}\left[\alpha_{1}\left(\omega_{1}\right), \delta n_{1}\left(\omega_{1}\right), \alpha_{2}\left(\omega_{2}\right), \delta n_{2}\left(\omega_{2}\right)\right],
\end{aligned}
$$

with $\delta n_{i}\left(\omega_{i}\right)=n_{i}\left(\omega_{i}\right)-1$, the deviation of the index of refraction from $1, \alpha_{i}\left(\omega_{i}\right)$ as the absorption coefficient and $\mathrm{l}$ as the length of the interaction zone. The 
absorption in the probe is given by the DC-signal, the dispersion is contained in the phase of the AC-signal. This phase depends also on the distance $L_{2}$ between the beamsplitter and PD2.

Connecting the AC-signal to the RF-entrance of mixer 2, the IF-output at the mixer is

$$
\begin{aligned}
I_{\mathrm{IF}}= & \frac{1}{2} \eta_{1} E_{10} E_{20} \cos \left[\left(\omega_{1}-\omega_{2}\right)\left(t+L_{1} / c\right)\right] \\
\times & \frac{1}{2} \eta_{2} E_{10} E_{20} \mathrm{e}^{-\left[\alpha_{1}\left(\omega_{1}\right)+\alpha_{2}\left(\omega_{2}\right)\right] l / 2} \\
\times & \cos \left\{\left(\omega_{1}-\omega_{2}\right)\left(t+L_{2} / c\right)\right. \\
& \left.\quad+\left[\delta n_{1}\left(\omega_{1}\right) \omega_{1}-\delta n_{2}\left(\omega_{2}\right) \omega_{2}\right] l / c\right\} \\
= & \eta_{1} \eta_{2} I_{10} I_{20} \mathrm{e}^{-\left\{\alpha_{1}\left(\omega_{1}\right)+\alpha_{2}\left(\omega_{2}\right) \mid l / 2\right.} \\
\times & \cos \left\{\left(\omega_{1}-\omega_{2}\right) \frac{L_{1}-L_{2}}{c}\right. \\
& \left.\quad+\left[\delta n_{1}\left(\omega_{1}\right) \omega_{1}-\delta n_{2}\left(\omega_{2}\right) \omega_{2}\right] l / c\right\},
\end{aligned}
$$

depending on the amplitude and phase-difference between both signals connected to this mixer.

Comparing this with the signal of a Mach-Zehnder interferometer:

$I_{\text {homodyne }} \propto E_{0}^{2} \mathrm{e}^{-\alpha l / 2}$

$$
\times \cos \left(\omega \frac{L_{1}-L_{2}}{c}+[\delta n(\omega) \omega l / c]\right),
$$

it becomes obviously clear that while changing the laser frequency the homodyne detector generates an additional signal proportional to the length difference $L_{1}-L_{2}$. The heterodyne detector produces this additional signal only if the laserscan is done by changing the difference frequency generated by the MS and not when the scan is done with fixed difference frequency.

One of the advantages of the heterodyne setup is that the noise induced by the unavoidable changes of the length difference $L_{1}-L_{2}$ caused by e.g. vibration or acoustic noise scale only with the difference frequency and not with the absolute frequency as in homodyne detectors. This indicates a noise reduction of more than four orders of magnitude compared with traditional interferometers. As the phase fluctuations of the difference frequency $\omega_{1}-\omega_{2}$ of phase locked lasers are usually smaller than the phase fluctuations of the frequency $\omega$ of a single laserfield the additional noise produced when $L_{1}-L_{2} \neq 0$ is smaller for hetcrodyne detectors than for homodyne detcctors.

If the second laser field does not interact with the atomic transitions $\left(\alpha_{2}\left(\omega_{2}\right)=0=\delta n_{2}\left(\omega_{2}\right)\right)$ the IF- signal depends only on the absorption and dispersion of the first field:

$$
\begin{aligned}
I_{\mathrm{IF}} & =\eta_{1} \eta_{2} I_{10} I_{20} \mathrm{e}^{-\alpha_{1}\left(\omega_{1}\right) l / 2} \\
& \times \cos \left(\left(\omega_{1}-\omega_{2}\right) \frac{L_{1}-L_{2}}{c}+\delta n_{1}\left(\omega_{1}\right) \omega_{1} l / c\right) \\
& =\eta_{1} \eta_{2} I_{10} I_{20} \mathrm{e}^{-\alpha_{1}\left(\omega_{1}\right) / / 2} \cos \left[\Phi+\delta n_{1}\left(\omega_{1}\right) \omega_{1} l / c\right],
\end{aligned}
$$

where $\Phi=\left(\omega_{1}-\omega_{2}\right)\left(L_{1}-L_{2}\right) / c$ is the offset phase described above.

The calibration (e.g. the determination of $\eta_{1} \eta_{2} I_{10}$ $\times I_{20}$ ) of this detector is very similar to the calibration of a homodyne detector. It can be done with no medium in the interaction zone or with detuned lasers, oscillating in the same difference frequency range $\left(\delta n_{1}\left(\omega_{1}\right)=0=\alpha_{1}\left(\omega_{1}\right)\right)$. For this calibration the length difference or the difference frequency has to be adjusted for $\Phi=N \times 2 \pi$, which corresponds to maximum $I_{\mathrm{IF}}$-signal. Together with the measured intensities $\eta_{2} I_{10}, \eta_{2} I_{20}$ the index of refraction can now be calculated from the $I_{\mathrm{DC}}$ - and the $I_{\mathrm{IF}}$-signal. For working with maximal slope efficiency the difference frequency or the length difference has to be changed again without changing the laser power on the photodetector, so that the $I_{\mathrm{IF}}$ vanishes, corresponding to $\Phi=N \times 2 \pi-\pi / 2$ :

$$
\begin{aligned}
& \cos \left[N \times 2 \pi-\pi / 2+\delta n_{1}\left(\omega_{1}\right) \omega_{1} l / c\right] \\
& \quad=\sin \left[\delta n_{1}\left(\omega_{1}\right) \omega_{1} l / c\right] .
\end{aligned}
$$

For small phase shifts the response of this setup is directly proportional to the change of the index of refraction:

$$
\begin{aligned}
& \delta n_{1}\left(\omega_{1}\right) \omega_{1} l / c \ll 1 \\
& \quad \rightarrow I_{\mathrm{IF}} \simeq \eta_{1} \eta_{2} I_{10} I_{20} \mathrm{e}^{-\alpha_{1}\left(\omega_{1}\right) l / 2} \delta n_{1}\left(\omega_{1}\right) \omega_{1} l / c .
\end{aligned}
$$

For FMS the comparable signal is insensitive to acoustic noise but scales only with the modulation index $M$ e.g. [8]:

$I(t) \propto \eta M I_{0} \delta n(\omega) \omega l / c$.

As the modulation index is small compared with unity, for a given total intensity the fundamental signal to shot noise limit is lower than for the heterodyne setup with equal intensities in both fields. 
Another often more important advantage of the heterodyne setup is that it is very useful for coherent two photon resonances. As the difference frequency of both fields has to be detected independently from the interaction region to phase lock both fields, only the beat behind the interaction region and an additional mixer is necessary to measure the dispersion of this coherent effect.

The probe can now be entered in the interaction region. Scanning the second laser over the interesting frequency range (the first laser will follow this scan because of the phase locked loop described above so that the difference frequency will not change) the dispersion and absorption can be calculated from the measured $I_{\mathrm{DC}}$ and $I_{\mathrm{IF}}$ signals:

$$
\begin{aligned}
& \alpha_{1}\left(\omega_{1}\right)=-\frac{1}{l} \ln \left(\frac{I_{\mathrm{DC}}\left(\omega_{1}\right)-\eta_{2} I_{20}}{\eta_{2} I_{10}}\right), \\
& \delta n_{1}\left(\omega_{1}\right)=\frac{c}{\omega_{1} l} \arcsin \left(\frac{I_{\mathrm{IF}}\left(\omega_{1}\right)}{\eta_{1} \eta_{2} I_{10} I_{20} \mathrm{e}^{-\alpha_{1}\left(\omega_{1}\right) l / 2}}\right) .
\end{aligned}
$$

One of the disadvantages of most techniques to measure the dispersion of an optical transition is that the calculated index of refraction depends on the measured absorption. This problem can be solved by connecting the AC-signals of the photodiodes to electronic comparators $\mathrm{EC}$ before heterodyning them with mixer 2 .

$$
\begin{aligned}
& U_{\mathrm{ECl}}=U_{0} \operatorname{sign}\left(I_{\mathrm{ACl}}\right)=U_{0} \\
& \quad \times \operatorname{sign}\left\{\cos \left[\left(\omega_{1}-\omega_{2}\right)\left(t+L_{1} / c\right)\right]\right\}, \\
& U_{\mathrm{EC} 2}=U_{0} \operatorname{sign}\left(I_{\mathrm{AC} 2}\right)=U_{0} \\
& \quad \times \operatorname{sign}\left\{\cos \left[\left(\omega_{1}-\omega_{2}\right)\left(t+L_{2} / c\right)+\delta n_{1}\left(\omega_{1}\right) l / c\right]\right\} .
\end{aligned}
$$

This would lead us to an $I_{\mathrm{IF}}$-signal which is independent from the absorption in the probe:

$$
I_{\mathrm{IF}}=U \sin \left[\delta n_{1}\left(\omega_{1}\right) l / c\right]+\mathrm{O}\left[\left(\omega_{1}-\omega_{2}\right) t\right] .
$$

Another advantage of using EC's is that the calibration of the signal is independent from laserpower.

The interesting optical properties of a coherent population trapping scheme examined in the last few years are the dispersion and absorption. The main attribute of a CPT-scheme is a large dispersion $\mathrm{d} n / \mathrm{d} w$, often described in terms of the group velocity

$l_{\mathrm{g}}=\frac{c}{n+\omega \times \mathrm{d} n / \mathrm{d} w}$

\section{Coherent Population Trapping}

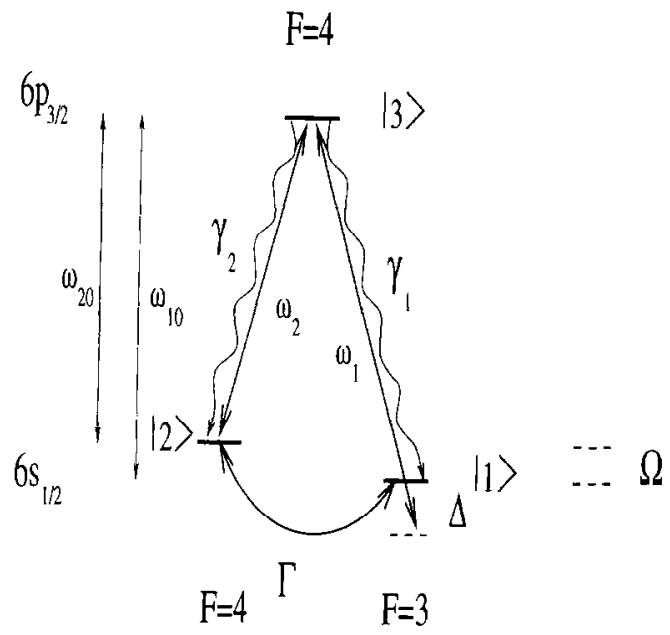

Fig. 2. Realization of the CPT-system in cesium: The groundstate hyperfine levels $\left(6 s_{1 / 2} F=3,4\right)$ are both coupled with one of the hyperfine levels $\left(6 p_{3 / 2} F^{\prime}=4\right)$ of the upper level of the $D_{2}$-line. The groundstate splitting is $\Omega=9.1926 \mathrm{~Hz}$. The first laser is detuned by $\Delta$ from the first transition $\left(\omega_{1}-\omega_{10}=\Delta\right)$. The second laser is on resonance with the second transition $\left(\omega_{2}=\omega_{20}\right)$. The decay rate $r$ of the coherence is determined by collisions. The spontaneous emission rates of the upper level are $\gamma_{1,2}$.

with nearly vanishing absorption. These properties are induced by a second laser field which fulfills a twophoton Raman resonance condition (see Fig. 2).

If the frequency $\omega_{1}$ of laser 1 is detuned by $\Delta_{1}$ from the first transition and $\omega_{2}$ detuned by $\Delta_{2}$ from the second transition, the refraction and absorption for both fields are $[1,3,6,11]$ :

$$
\begin{aligned}
& n_{1}\left(\Delta_{1}, \Delta_{2}\right)=1+\frac{3}{8 \pi^{2}} \gamma_{1} \lambda^{3} N \frac{\left(\Delta_{1}-\Delta_{2}\right) A_{1}-\frac{1}{2} \Gamma B_{1}}{A_{1}^{2}+B_{1}^{2}}, \\
& n_{2}\left(\Delta_{1}, \Delta_{2}\right)=1+\frac{3}{8 \pi^{2}} \gamma_{2} \lambda^{3} N \frac{\left(\Delta_{2}-\Delta_{1}\right) A_{2}-\frac{1}{2} \Gamma B_{2}}{A_{2}^{2}+B_{2}^{2}}, \\
& \alpha_{1}\left(\Delta_{1}, \Delta_{2}\right)=\frac{3}{4 \pi} \lambda^{2} N \gamma_{1} \frac{\frac{1}{2} \Gamma A_{1}+\left(\Delta_{1}-\Delta_{2}\right) B_{1}}{A_{1}^{2}+B_{1}^{2}} \\
& \alpha_{2}\left(\Delta_{1}, \Delta_{2}\right)=\frac{3}{4 \pi} \lambda^{2} N \gamma_{2} \frac{\frac{1}{2} \Gamma A_{2}+\left(\Delta_{2}-\Delta_{1}\right) B_{2}}{A_{2}^{2}+B_{2}^{2}}
\end{aligned}
$$

where $\gamma_{i}$ are the linewidth of the single transitions, $\Gamma \ll \gamma_{i}$ the inverse lifetime of the coherence normally dominated by collisions, $\lambda$ is the wavelength of the 
laser fields and $N$ the atomic density in the interaction zone. $A_{i}$ and $B_{i}$ are defined as:

$A_{i}:=S \Omega_{\mathrm{R}}^{2}+\frac{1}{4} \Gamma\left(\gamma_{1}+\gamma_{2}\right)-\Delta_{i}\left(\Delta_{i}-\Delta_{j}\right)$,

$B_{i}:=\frac{1}{2}\left(\left(\Delta_{i}-\Delta_{j}\right)\left(\gamma_{1}+\gamma_{2}\right)+\Delta_{i} \Gamma\right), \quad(i \neq j)$,

with $\Omega_{R}=\sqrt{\Omega_{1}^{2}+\Omega_{2}^{2}}$ as an effective Rabi frequency. For sufficiently large Rabi frequencies $\Omega_{\mathrm{R}}^{2}>\frac{1}{4} \Gamma\left(\gamma_{1}+\right.$ $\gamma_{2}$ ) and small detunings $\Delta_{1,2}<\Omega_{1,2}$ these coefficients can be approximated by

$A_{i} \simeq \Omega_{\mathrm{R}}^{2}, \quad B_{i} \simeq\left(\Delta_{i}-\Delta_{j}\right) \gamma$,

with an effective linewidth of $\gamma=\frac{1}{2}\left(\gamma_{1}+\gamma_{2}\right)$. With these approximations the deviation of the index of refraction from 1 depends linearly on the detunings of both fields and the absorption vanishes on the two photon resonance:

$\delta n_{1}\left(\Delta_{1}, \Delta_{2}\right) \simeq \frac{3}{8 \pi^{2}} \gamma_{1} \lambda^{3} N \frac{\Delta_{1}-\Delta_{2}}{\Omega_{\mathrm{R}}^{2}}$

$\delta n_{2}\left(\Delta_{1}, \Delta_{2}\right) \simeq \frac{3}{8 \pi^{2}} \gamma_{2} \lambda^{3} N \frac{\Delta_{2}-\Delta_{1}}{\Omega_{\mathrm{R}}^{2}}$,

$\alpha_{1}\left(\Delta_{1}, \Delta_{2}\right) \simeq \frac{3}{4 \pi} \lambda^{2} N \gamma_{1}\left(\frac{\frac{1}{2} \Gamma}{\Omega_{\mathrm{R}}^{2}}+\frac{\left(\Delta_{1}-\Delta_{2}\right)^{2} \gamma}{\Omega_{\mathrm{R}}^{4}}\right)$,

$\alpha_{2}\left(\Delta_{1}, \Delta_{2}\right) \simeq \frac{3}{4 \pi} \lambda^{2} N \gamma_{2}\left(\frac{\frac{1}{2} \Gamma}{\Omega_{\mathrm{R}}^{2}}+\frac{\left(\Delta_{1}-\Delta_{2}\right)^{2} \gamma}{\Omega_{\mathrm{R}}^{4}}\right)$.

In our experimental setup two identical laserdiodes in a Hollberg-setup [12] were used and tuned to the $\mathrm{D}_{2}$ absorption line of $\mathrm{Cs}(\lambda=852 \mathrm{~nm})$. Both lasers were superimposed at the beamsplitter. The first output was used to detect the beat signal as described above. One part of this signal was connected to the LO-entrance of mixer 2. The other part was used to phase lock both lasers on a tunable difference frequency generated by the MS $\omega_{1}=\omega_{2}+\nu_{\mathrm{MS}}$, so that laser I could be scanned by tuning the MS. As the measurement of the linewidth of the beat signal was limited by the minimal resolution bandwidth of the spectrum analyzer of $3 \mathrm{~Hz}$, we can only give this upper limit for the linewidth of the difference frequency.

The second output of the beamsplitter was focused into a single mode fiber to achieve a good overlap of the transversal modes of both lasers. This mode cleaned superimposed light went through the probe with a diameter of the laserbeams in the interaction zone of about $8 \mathrm{~mm}$ to reduce transit time broadening to about $\approx 50 \mathrm{kHz}$.

The medium was a Cs-atomic beam and we measured the dispersion and absorption of a CPT-scheme created between the ground state hyperfine splitting $\left(6 s_{1 / 2} F=3,4\right)$ and one of the hyperfine states $\left(6 p_{3 / 2} F^{\prime}=4\right)$ of the upper level of the $\mathrm{D}_{2}$ absorption line (see Fig. 2). The difference frequency between hoth transitions is $\Omega=\omega_{10}-\omega_{20}=2 \pi \times 9.192631$ $\mathrm{GHz}$. The linewidths of the optical transitions are $\gamma_{1} \simeq \gamma_{2} \simeq 5 \mathrm{MHz}$. The frequency of laser 2 is $\omega_{2}$ and $\omega_{1}$ is the frequency of laser 1 detuned by $\Delta$ from the two photon resonance. In the interaction region the atomic beam has a density of about $10^{9} / \mathrm{cm}^{3}$ and a width of $l=1 \mathrm{~cm}$ with a residual Doppler broadening of $\delta \nu$ (FWHM) $\simeq 40 \mathrm{MHz}$ for the optical transitions or $\simeq 1 \mathrm{kHz}$ for the CPT-resonance. The inverse lifetime $\Gamma<100 \mathrm{~Hz}$ of the coherence is determined by collisions in the atomic beam and was estimated from the density, the velocity and the collision cross section [13] of the atoms in the interaction region.

In the measurements presented here the second laser is also used as the reference beam. This means that in contrast to the technique described above the dispersion and absorption of the reference beam can now not be neglected.

If the dispersion and absorption of both fields depend on the detuning $\Delta$ like in a CPT scheme these properties can only be measured simultaneously. For investigating the effects on the two fields separately this problem could be solved by inducing a third laser field with a frequency detuned from all atomic transitions as described above or using FMS-techniques. Another possibility is a pump-probe experiment with one strong pumpfield and a weak probefield, neglecting the modification of the pumpfield induced by the probefield. But as most applications of these coherent effects are only interested in the induced phaseshift like in a magnetometer or a gravitational wave detector [3.4], the signal strength is important. Maximal signal strength is achieved using equal power at equal Rabi-frequencies.

The powers of both fields were $I_{10} \simeq I_{20} \simeq 200$ $\mu \mathrm{W}$, respectively. This leads to an effective Rabi frequency of $\Omega_{\mathrm{R}} \simeq 500 \mathrm{kHz}$.

So for the results presented here we assume: 
$\alpha_{1}\left(\Delta_{1}, \Delta_{2}\right) \approx \alpha_{2}\left(\Delta_{1}, \Delta_{2}\right)=: \alpha(\Delta)$,

$\delta n_{1}\left(\Delta_{1}, \Delta_{2}\right) \approx-\delta n_{2}\left(\Delta_{1}, \Delta_{2}\right)=: \delta n(\Delta)$.

These approximations inserted in (2) lead to an $I_{\mathrm{DC}}$ signal:

$I_{\mathrm{DC}}(\Delta)=\eta_{2}\left(I_{10}+I_{20}\right) \mathrm{e}^{-\alpha(\Delta) l}=\eta_{2} I_{0} \mathrm{e}^{-\alpha(\Delta) l}$,

with $I_{0}=I_{10}+I_{20}$ as the total power. The $I_{\mathrm{IF}}$ signal could be approximated by:

$$
\begin{aligned}
& I_{\mathrm{IF}}(\Delta)=\eta_{1} \eta_{2} I_{0}^{2} \mathrm{e}^{-\alpha(\Delta) l} \\
& \quad \times \cos \left[(\Omega+\Delta)\left(L_{1}-L_{2}\right) / c+\delta n(\Delta)\left(\omega_{1}+\omega_{2}\right) l / c\right] .
\end{aligned}
$$

As the scan of laser 1 was done by scanning the MS over the difference frequency of both transitions, the (now frequency dependent) residual phaseshift $\Phi(\Delta)=(\Omega+\Delta)\left(L_{1}-L_{2}\right) / c$ has to be calibrated. This calibration was done with detuned lasers, oscillating in the same difference frequency range. Also the amplitude $\eta_{1} \eta_{2} I_{0}^{2}$ of the $I_{\mathrm{IF}}$ signal and the amplitude $\eta_{2} I_{0}$ of the $I_{\mathrm{DC}}$-signal were measured. After this calibration the second laser was roughly tuned to the Doppler broadened resonance with the second transition ( $F=4 \leftrightarrow F^{\prime}=4$ ) controlled by the fluorescence of the atomic beam (this leads to a frequency offset from the atomic resonance frequency $\leq 1 \mathrm{MHz}$, which is much lower than the residual Doppler broadening in the atomic beam of $40 \mathrm{MHz}$ ). The first laser was heterodyne phaselocked with a difference frequency of $\nu_{\mathrm{MS}}=\Omega+\Delta$ generated by the MS. By scanning the MS over the interesting frequency range, the frequency of laser 1 was tuned over the first transition $\left(F=3 \leftrightarrow F^{\prime}=4\right)$.

In consideration of the residual phaseshift the $a b-$ sorption and dispersion was calculated from these signals:

$$
\begin{aligned}
& \alpha(\Delta)=-\frac{1}{l} \ln \left(\frac{I_{\mathrm{DC}}(\Delta)}{\eta_{2} I_{0}}\right), \\
& \delta n(\Delta)=\frac{c}{\left(\omega_{1}+\omega_{2}\right) l} \\
& \quad \times\left[\arccos \left(\frac{I_{\mathrm{IF}}(\Delta)}{\eta_{1} \eta_{2} I_{0}^{2} \mathrm{e}^{-\alpha(\Delta) l}}\right)-(\Omega+\Delta)\left(L_{1}-L_{2}\right)\right] .
\end{aligned}
$$
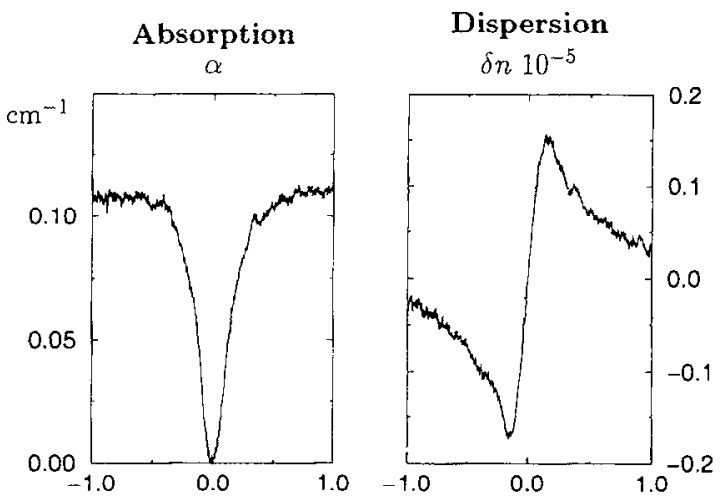

Fig. 3. The left graphs show the absorption coefficient $\alpha$, the right graphs the deviation $\delta n(\Delta)$ of the index of refraction from 1 in the atomic beam in dependence from the two-photon detuning $\Delta$ in $\mathrm{MHz}$. The dispersion corresponds to a group velocity of $v_{\mathrm{g}}=c / 5000$.

The right part of Fig. 3 shows the absorption coefficient, the left part the deviation of the index of refraction from 1 as a function of the detuning from the two photon resonance. The linewidth (peak to peak of the dispersion line $\simeq$ FWHM of absorption line) is 300 $\mathrm{kHz}$. The dispersion in the center of the line corresponds to a group velocity of $v_{\mathrm{g}} \simeq c / 5000$. The signal to noise ratio was determined by electronic noise.

As the approximations used above are only allowed for small detunings [3] $\left(\Delta_{1,2}<\Omega_{1}, \Omega_{2}\right)$ from the CPT-resonance, the index of refraction of field 1 should differ from the index of refraction of field 2 at difference frequencies larger than the Rabi frequencies. This leads to a larger dispersion for one of the fields and a lower dispersion for the other one as the calculated one.

In this lelter we presented a new method to measure the dispersion and the absorption of an atomic transition. This method is more than four orders of magnitude less sensitive to acoustic noise or vibrations than the traditional homodyne detectors. Compared with the frequency modulation spectroscopy the heterodyne interferometer setup has a $1 / M$ higher signal strength at the same shot noise level, but more important it is easy to use for coherent two photon spectroscopy. Results are presented from the first investigations of the dispersion and absorption of a coherent population trapping scheme in a Cs atomic beam measured with this new technique. 


\section{References}

1 1 P.M. Radmore. P.L. Knight, J. Phys. B. 15 (1982) 561.

$12 \mid$ M.O. Scully, S.Y. Zhu, H. Fearn, Z. Phys. D 22 (1992) 471.

13| M.O. Scully, M. Fleischhauer, Phys. Rev. Lett. 69 (1992) 1360.

|4| M. Fleischhauer, K. Danzmann. The length amplifier. in preparation.

15| M. Xian, Y. I.i, S Jin, J. Gea-Banacloche, Phys. Rev. Lett. 74 (1995) 666

$16 \mid$ O. Schmidt, R. Wynands, Z. Hussein, D. Meschede, Phys. Rev. A 53 (1996) R27.

17| H.R. Gray, R.M. Whitley, C.R. Stroud Jr., Optics Lett. 3 (1978) 218
[8] G.C. Bjorklund, Optics Lett. 5 (1980) 15.

[9] J.L. Hall, L. Hollberg. T. Baer, H.G. Robinson, Appl. Phys. Lett. 39 (1981) 680.

[10] J.L. Hall, M. Long-Sheng. G. Kramer, IEEE J. Quantum Electron. 23 ( 1987) 427

[11] B.W. Shore. The theory of coherent atomic excitation (Wiley, New York, 1990).

(12) P. Laurent, A. Clairon, C. Breant, IEEE J. Quantum Electron. 25 (1989) 1131 .

[13] I. Estermann, S.N. Foner, O. Stern, Phys. Rev. 71 (1947) 250. 